\title{
ACCESS - A Concept Study for the Direct Imaging and Spectroscopy of Exoplanetary Systems
}

\author{
John Trauger ${ }^{\mathrm{a}}$, Karl Stapelfeldt ${ }^{\mathrm{a}}$, Wesley Traub ${ }^{\mathrm{a}}$, John Krist ${ }^{\mathrm{a}}$, Dwight Moody ${ }^{\mathrm{a}}$, Dimitri Mawet ${ }^{\mathrm{a}}$ \\ Eugene Serabyn ${ }^{\mathrm{a}}$, Curt Henry ${ }^{\mathrm{a}}$, Paul Brugarolas ${ }^{\mathrm{a}}$, James Alexander ${ }^{\mathrm{a}}$, Robert Gappinger ${ }^{\mathrm{a}}$ \\ Olivia Dawson ${ }^{\mathrm{a}}$, Virgil Mireles ${ }^{\mathrm{a}}$, Peggy Park ${ }^{\mathrm{a}}$, Laurent Pueyo ${ }^{\mathrm{a}}$, Stuart Shaklan $^{\mathrm{a}}$ \\ Olivier Guyon ${ }^{\mathrm{b}, \mathrm{c}}$, Jeremy Kasdin ${ }^{\mathrm{d}}$, Robert Vanderbei ${ }^{\mathrm{d}}$, David Spergel ${ }^{\mathrm{d}}$, Ruslan Belikov ${ }^{\mathrm{e}}$ \\ Geoff Marcy $^{\mathrm{f}}$, Robert A. Brown ${ }^{\mathrm{g}}$, Jean Schneider ${ }^{\mathrm{h}}$, Bruce Woodgate ${ }^{\mathrm{i}}$, Robert Egerman ${ }^{\mathrm{j}}$ \\ Gary Matthews ${ }^{j}$, Jason Elias ${ }^{j}$, Yves Conturie ${ }^{j}$, Phillip Vallone ${ }^{j}$, Perry Voyer ${ }^{j}$, Ronald Polidan ${ }^{k}$ \\ Chuck Lillie $^{\mathrm{k}}$, Constance Spittler ${ }^{\mathrm{k}}$, David Lee ${ }^{\mathrm{k}}$, Reem Hejal ${ }^{\mathrm{k}}$, Allen Bronowicki ${ }^{\mathrm{k}}$, Nick Saldivar ${ }^{\mathrm{k}}$ \\ Mark Ealey ${ }^{\mathrm{m}}$, Thomas Price ${ }^{\mathrm{m}}$ \\ ${ }^{a}$ Jet Propulsion Laboratory,California Institute of Technology, Pasadena, CA 91109 \\ ${ }^{\mathrm{b}}$ Subaru Telescope, Hilo, HI 96720 \\ ${ }^{c}$ University of Arizona, Tucson, AZ 85721 \\ ${ }^{\mathrm{d}}$ Princeton University, Princeton, NJ 08540 \\ ${ }^{e}$ NASA Ames Research Center, Moffett Field, CA 94035 \\ ${ }^{\mathrm{f}}$ University of California, Berkeley, CA 94720 \\ ${ }^{g}$ Space Telescope Science Institute, Baltimore, MD 21218 \\ ${ }^{\mathrm{h}}$ Paris Observatory, Meudon, France \\ ijASA Goddard Space Flight Center, Greenbelt, MD 20771 \\ ${ }^{\mathrm{j}}$ ITT Space Systems Division, Rochester, NY 14650 \\ ${ }^{\mathrm{k}}$ Northrop Grumman Corporation, Redondo Beach, CA 90278 \\ ${ }^{\mathrm{m}}$ Northrop Grumman Xinetics, Devens, MA 01434
}

\begin{abstract}
ACCESS is one of four medium-class mission concepts selected for study in 2008-9 by NASA's Astrophysics Strategic Mission Concepts Study program. ACCESS evaluates a space observatory designed for extreme high-contrast imaging and spectroscopy of exoplanetary systems. An actively-corrected coronagraph is used to suppress the glare of diffracted and scattered starlight to contrast levels required for exoplanet imaging. The ACCESS study considered the relative merits and readiness of four major coronagraph types, and modeled their performance with a NASA medium-class space telescope. The ACCESS study asks: What is the most capable medium-class coronagraphic mission that is possible with telescope, instrument, and spacecraft technologies available today? Using demonstrated high-TRL technologies, the ACCESS science program surveys the nearest 120+ AFGK stars for exoplanet systems, and surveys the majority of those for exozodiacal dust to the level of 1 zodi at $3 \mathrm{AU}$. Coronagraph technology developments in the coming year are expected to further enhance the science reach of the ACCESS mission concept.
\end{abstract}

Keywords: coronagraphs, exoplanets, space astronomy, active optics

\section{INTRODUCTION}

Our science objective is the direct observation of exoplanetary systems, possibly dynamically full, that harbor exoplanets, planetesimals, and dust/debris structures. Direct coronagraphic imaging at visible (450-900 nm) wavelengths and low-resolution $(\mathrm{R}=20)$ spectroscopy of exoplanet systems in reflected starlight enables a broad science program that includes a census of nearby known radial velocity $(\mathrm{RV})$ planets in orbits beyond $\sim 1 \mathrm{AU}$; a search for mature exoplanet systems beyond the RV survey limits including giant planets, super-earths, and possibly a dozen earth-mass planets; observations of debris structures as signposts of unseen planets and planetesimals; and imaging of dust

Space Telescopes and Instrumentation 2010: Optical, Infrared, and Millimeter Wave, edited by Jacobus M. Oschmann Jr., Mark C. Clampin, Howard A. MacEwen, Proc. of SPIE Vol. 7731, 773128 - (c) 2010 SPIE · CCC code: 0277-786X/10/\$18 - doi: 10.1117/12.858301 
structures in circumstellar environments as a probe of the life cycle of planetary systems from young stellar objects to proto-planetary nebulae.

The ACCESS study ${ }^{1}$ compares the performance and readiness of four major coronagraph architectures. ACCESS defines a conceptual space observatory platform as the "level playing field" for comparisons among coronagraph types. And it uses laboratory validation of these representative coronagraph types as a second "level playing field" for assessing coronagraph hardware readiness. The external occulter coronagraph is not considered here, on the presumption that a concept requiring two formation-flying spacecraft is beyond the bounds of a medium-class mission. ACCESS defines a genre of scientifically compelling mission concepts built upon mature subsystem technologies, and evaluates the science reach of a medium-class coronagraph mission.

In Section 2, we review the science investigations enabled by the ACCESS mission. The observatory and coronagraph architectures, as well as the computational methods leading to the predicted science program, are briefly described in Section 3.

\section{SCIENCE OBJECTIVES}

The architecture of exoplanetary systems, including planets and debris structures, lies beyond the reach of conventional techniques. The proposed exoplanet and zodi targets are too faint and too close to the parent star to be observable by current observatories on the ground or in space. RV and astrometry techniques are insensitive to dust structures, hence will not identify asteroid belts nor characterize the exozodiacal background levels. Transit and micro-lensing techniques, both dependent on chance alignments, provide statistical information on the prevalence of planets orbiting distant stars. Combined light spectroscopic methods are restricted to close-in giant planets. The contrast performance of large ground-based telescopes with adaptive optics currently falls orders of magnitude short of what is needed to image mature exoplanets or tenuous exozodiacal dust in reflected light around nearby stars.

ACCESS is designed to reveal planets and dust structures by reducing the scattered and diffracted light to within a few hundred milliarcseconds of a star at a level three orders of magnitude lower than any current instrument in space or on the ground, using a stable 1.5 meter diameter telescope, a coronagraphic system for control of diffracted light, active wavefront correction and suppression of scattered light, and a low-noise CCD focal plane and integral field spectrograph viewing the target systems in multiple spectral bands simultaneously.

\subsection{ACCESS exoplanet discovery space}

The sensitivities of various current and future techniques for the detection of exosolar planets are compared in Figure 1. Here asterisks indicate exoplanets identified by recent observations; curves indicate the detection thresholds for a variety of observatories, and blobs indicate the locus for Jupiter and Earth twins orbiting the nearest 100 AFGK stars.

Recent direct exoplanet detections are plotted in the diagram: Fomalhaut $b^{2,3}$, HR8799 b,c, $d^{4,5}$ and $\beta$-Pictorus $b^{6}$. The former has demonstrated the ability of a space coronagraph to detect an exoplanet with brightness relative to the star comparable to a mature Jupiter in the ACCESS target list, but at star-planet separations $\sim 60$ times greater than the inner working angle (IWA) for the ACCESS concept. The latter two detections indicate that the best demonstrated contrast in the infrared from ground-based observatories resides a factor of $\sim 10^{6}$ above the contrast floor for the ACCESS concept. It is evident that the location of the ACCESS sensitivity curve in the diagram sets the stage for a compelling science mission.

While Jupiter-twins orbiting the nearest 100 AFGK stars fall well within the ACCESS detection zone, Earth-twins and super-earths (up to twice the diameter of Earth) orbiting the same stars are near the margins. As illustrated in Figure 1, contrasts in the range of $10^{-7}$ to $10^{-8}$, yielding luminous young Jupiters, will be accomplished in the foreseeable future only by very large ground-based systems, assuming that these work as hoped, but the region from $10^{-8}$ to $10^{-10}$, where the mature Jupiters, super-earths, and dust disks reside, will be completely unknown territory until ACCESS or its equivalent flies in space. 


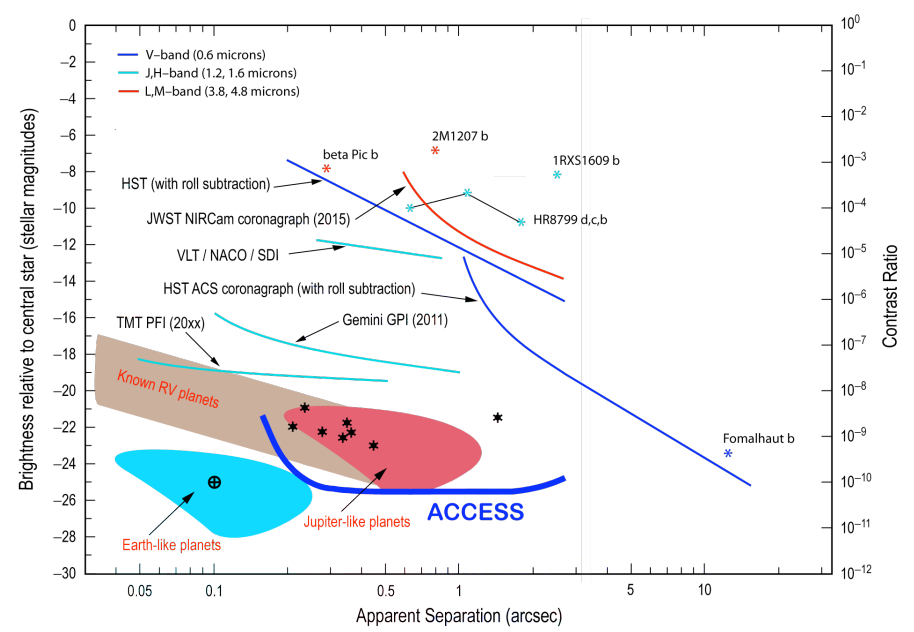

Figure 1. ACCESS imaging sensitivity compared to other methods available now and in the coming decade. Known exoplanets are indicated by asterisks. Curves indicate detection thresholds for representative groundbased and space observatories. Shaded areas indicate the regions of high probability (from Monte-Carlo simulations) of detectable planets orbiting the nearest 100 AFGK stars. Jupiters in 5 AU orbits fall in the redshaded area. Earth-like planets at $1 \mathrm{AU}$ fall in the blue-shaded area. The location of an Earth-twin at elongation at $10 \mathrm{pc}$ is indicated by the $\oplus$ symbol. The detection range for ACCESS is the area above the indicated bold curve.

\subsection{Spectra of known RV planets beyond $2 \mathrm{AU}$}

Radial velocity (RV) surveys have singled out the nearby stars that host Jovian planets. Ten nearby stars are currently known to host RV planets whose apastron distances project to angular separations greater than the ACCESS inner working angle of $3 \lambda / \mathrm{D}(0.23$ arcseconds at $\lambda=540 \mathrm{~nm})$. These are cold objects with a contrast of $10^{-9}$ in the optical and near-IR, and thus are only accessible to a space mission. Existing ephemerides allow observations to be timed to coincide with their maximum elongations, as indicated by the diagram at right. More than a dozen additional stars within $20 \mathrm{pc}$ show long-term accelerations indicating the presence of outer planets with orbits still not yet defined. ACCESS will directly detect these giant planets, measure their colors, take spectra, and provide astrometry that will resolve the sin $i$ ambiguity in their masses. The spectra will allow the strength of $\mathrm{CH}_{4}$ features to be measured and the depth of the uppermost cloud deck to be inferred. The planet's albedo and presence of bright ring systems can be inferred using the observed photometry and planet sizes estimated from their measured masses. Multi-epoch imaging showing the planetary orbital motions will make a powerful impression of the reality of exoplanetary systems in the public mind.

\subsection{Planet searches beyond RV survey limits}

RV searches to date have yielded detections of giant planets within 6 AU of (primarily) FGK stars with low chromospheric activity. Planets with longer periods or face-on orbits, and those orbiting active stars or A stars that lack a rich absorption spectrum, will escape RV detection. Two epochs of ACCESS imaging can detect objects to within 3 AU for stars within $15 \mathrm{pc}$, and inward to $1 \mathrm{AU}$ for perhaps a dozen targets. The stability of the ACCESS telescope will allow exoplanet detections down to contrasts of $10^{-10}$, corresponding to the brightness of a giant planet at 15 AU separation. Among the eighteen young stars (age less than 1 Gyr) within 20 pc, ACCESS can detect any companions greater than 5 Jupiter masses in their thermal emission in its longest-wavelength band.

For searches of the 3-15 AU region, 3 epochs of observations spaced at the beginning, middle, and end of a five-year mission will provide a sufficient exploration of orbital phase space to achieve a detection completeness exceeding $70 \%$. For the dozen stars where searches as close as $1 \mathrm{AU}$ are possible, 6-10 epochs of imaging may be needed to compile good completeness results. The newly discovered planets will then be characterized spectroscopically. For a handful of the brightest and nearest stars, ACCESS performance may be good enough to detect terrestrial planets in the habitable zone (HZ), for low levels of exozodiacal dust.

We have computed a simple metric as an indication of the search sensitivities with various coronagraph options. Using simulated coronagraph images, computed according to the observatory performance models described in the next section, and subjected to a Monte Carlo set of pointing errors predicted for the ACCESS observatory, we compute the 
number of fiducial Jupiters that could be observed at $45^{\circ}$ and $15^{\circ}$ from elongation at six epochs, with $\mathrm{S} / \mathrm{N}=10$ detections in V-band at each epoch, for a 5 -year mission in which $40 \%$ of the observing time is allocated to planet searches. The time needed to observe each target is subtracted from a pool of 12,264 hours ( 2 years with $70 \%$ observing efficiency) until the allocated pool of time is used up. The first example, in the first row of Table 1.1, corresponds to the laboratorydemonstrated Lyot coronagraph with an IWA $=3 \lambda / \mathrm{D}$, which we define as the performance floor for ACCESS coronagraphs. Highlighted in blue are tabulations for other coronagraph configurations, each of which corresponds to a theoretically possible demonstration of raw laboratory contrast of $10^{-9}$ or better, $20 \%$ spectral bandwidth, and inner working angles of $3 \lambda / \mathrm{D}$ or closer, all of which may be achieved in the foreseeable future. Planets appear at or beyond the $45^{\circ}$ separations from their stars at least half the time, therefore a sequence of six observing epochs spread across several years provides an observational completeness approaching $100 \%$.

Tables 1.1-3. A tabulation of the number of nearby stars that could be searched with various ACCESS coronagraphs to the depth of $10-\sigma$ detections of Jupiter twins in each of six visits to the star over a period of two years. The probability that an exoplanet will have a star-planet separation greater than that at $45^{\circ}$ elongation is $50 \%$ or more. Entries in the first row of Table 1.1 correspond to the coronagraph performance measured in the laboratory at 3.0 $\lambda / \mathrm{D}$ with the Lyot coronagraph as part of the ACCESS study. The other rows (highlighted in blue) represent coronagraph performance that may be achieved with further development of known technologies in the near future. The column for $45^{\circ}$ from maximum elongation corresponds to an observational completeness of $50 \%$ or more in each visit, approaching $100 \%$ after six epochs spread over several years.

Table 1.1. The number of nearby stars that can be surveyed for 5.2 AU Jupiters with an IWA $=3.0 \lambda / \mathrm{D}$

\begin{tabular}{|c|c|c|}
\hline Coronagraph Type & Planet $\mathbf{4 5}^{\circ}$ from max elong & Planet $\mathbf{1 5}^{\circ}$ from max elong \\
\hline Lyot & 117 & 175 \\
\hline PIAA & 166 & 278 \\
\hline Vortex & 135 & 204 \\
\hline
\end{tabular}

Table 1.2. The number of nearby stars that can be surveyed for 5.2 AU Jupiters with an IWA $=2.5 \lambda / \mathrm{D}$

\begin{tabular}{|c|c|c|}
\hline Coronagraph Type & Planet $\mathbf{4 5}^{\circ}$ from max elong & Planet $\mathbf{1 5}^{\circ}$ from max elong \\
\hline Lyot & 153 & 218 \\
\hline PIAA & 178 & 278 \\
\hline Vortex & 154 & 228 \\
\hline
\end{tabular}

Table 1.3. The number of nearby stars that can be surveyed for $5.2 \mathrm{AU}$ Jupiters with an IWA $=2.0 \lambda / / \mathrm{D}$

\begin{tabular}{|c|c|c|}
\hline Coronagraph Type & Planet $\mathbf{4 5}^{\circ}$ from max elong & Planet $\mathbf{1 5}^{\circ}$ from $\mathbf{~ m a x ~ e l o n g ~}$ \\
\hline Lyot & 170 & 230 \\
\hline Vortex & 164 & 241 \\
\hline
\end{tabular}

Another way to illustrate these data is shown in Figure 2. At left, the individual stars in the list are binned by observing time to reach $\mathrm{S} / \mathrm{N}=10$ detections, and plotted against distance and brightness of the central star. Another metric for ACCESS sensitivities is shown in Figure 2 at right. Computed in the same manner as Tables 1 above, the diagram indicates the number of detections of fiducial planets with the size and albedos of Jupiter, Saturn, Uranus, and superearths (with twice the diameter of earth) at $\mathrm{S} / \mathrm{N}=10$ in integration times of 1 day or less, as a function of distance from the star. Exozodiacal dust at the one-zodi equivalent level is assumed. For this simulation, we used the Lyot coronagraph, operating with an IWA of $2.5 \lambda / \mathrm{D}$. 

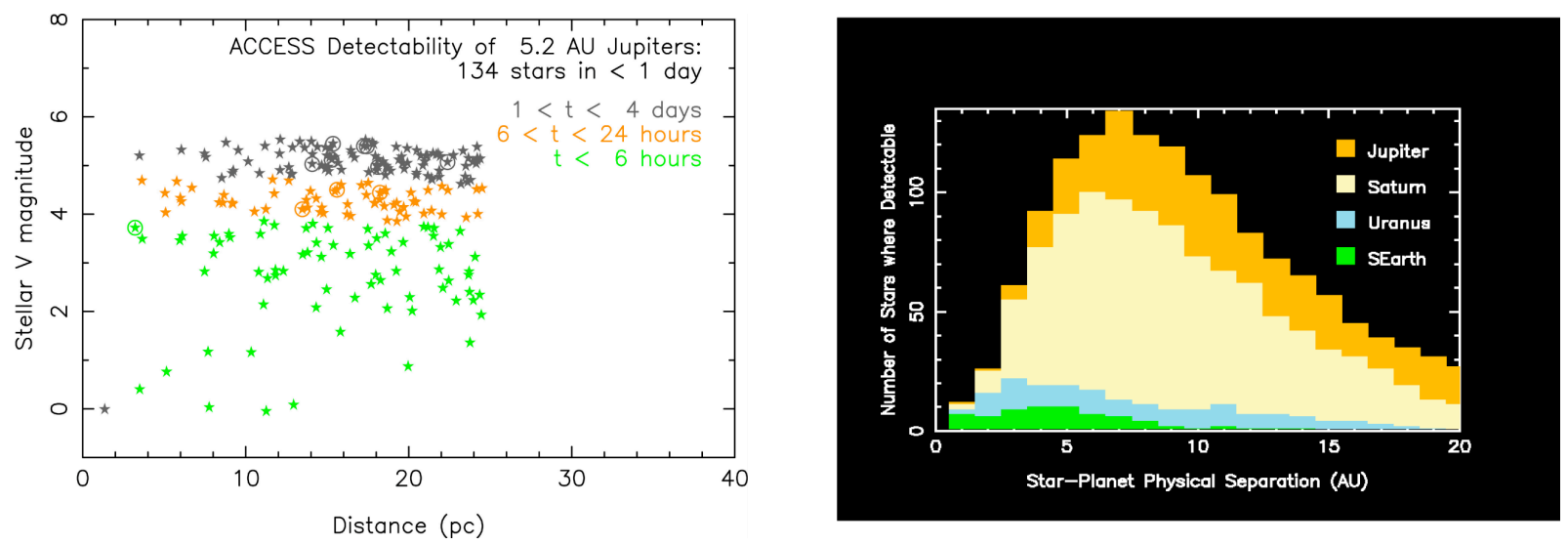

Figure 2. Two representations of the science reach in an ACCESS survey for exoplanets. At left are the detections of Jupiter-twins within $45^{\circ}$ of elongation from their parent stars, to $\mathrm{S} / \mathrm{N}=10$, using the ACCESS Lyot coronagraph with an IWA $=2 \lambda \mathrm{D}$ for a number of integration times. Note that the probability that an exoplanet will have a star-planet separation greater than that at $45^{\circ}$ elongation is $50 \%$ or more. At right, the number of planets, in four mass categories, detectable to $\mathrm{S} / \mathrm{N}=10$ in integration times of one day or less, using the ACCESS Lyot coronagraph with an IWA of $2.5 \lambda / \mathrm{D}$.

\subsection{Zodi structure as an indicator of unseen planets}

ACCESS will make the most sensitive imaging survey to date of tenuous debris disks, the signposts of planetary systems. These are clouds of small dust grains created by the mutual collisions of asteroids and comets, as well as by comet sublimation. Without continual replenishment by such events, the dust would be cleared away within a small fraction of a star's lifetime by stellar radiation, stellar wind pressure, or self-radiation. The interplanetary dust cloud in our own solar system, which can be seen as the zodiacal light, is a debris disk created primarily by the collisions of asteroids. Spitzer observations have identified more than 80 stars within $25 \mathrm{pc}$ that possess debris disk analogs to our Kuiper Belt at the levels of a few hundred zodis. Some of these dust clouds have been spatially resolved and show warps, asymmetries, or narrow rings that are strongly suggestive of planetary perturbations. The structure of the eccentric, sharp-edged Fomalhaut debris ring demanded a nearby planetary perturber which was spectacularly confirmed by recent HST observations ${ }^{3}$. Hundreds of nearby stars are likely to have debris disks that can be imaged with ACCESS contrast capabilities at $1 \mathrm{AU}$ resolution. As a canvas on which planets can impress dynamical signatures, dust disk structures will enable indirect detections of numerous planets too faint or too distant to be discovered any other way.

\subsection{Probe of Zodi dust as a potential problem for terrestrial planet detection}

For all telescope architectures, high levels of exozodiacal dust will be a major impediment to direct imaging of extrasolar terrestrial planets. These dust clouds reflect starlight and emit in the thermal infrared, adding photon noise and scene confusion to exoplanet signals. It is therefore crucial to assess the dust levels around nearby stars. The Keck and LBT interferometers use $10 \mu \mathrm{m}$ nulling observations to probe $\mathrm{HZ}$ dust. However, atmospheric and instrumental phase instability has limited the achievable null depth thus far to sensitivities of roughly a hundred zodis. Additional ways to attack the problem are needed. Detailed coronagraphic imaging simulations show that ACCESS can provide sensitivity down to the sub-zodi level, extending inward to $3 \mathrm{AU}$ across the full target sample envisaged for a Terrestrial Planet Finder (TPF) mission, and inward to 1 AU for about a dozen stars. Transport models can be used to relate dust levels at 3 AU to those at $1 \mathrm{AU}$. For our solar system, these models are well-calibrated by dust counts from interplanetary probes. Reflected light observations with ACCESS will thus be an outstanding tool for assessing the dust content in the HZs of nearby stars.

\subsection{General astrophysics with ACCESS}

Extending a thousand times beyond the performance of HST, the contrast capabilities of ACCESS will enable groundbreaking studies of circumstellar matter and quasar/AGN host galaxies.

For protostellar and protoplanetary disks, thousands of targets are known within 500 pc from IRAS and Spitzer surveys, but very few have been detected against the glare of their central star. Imaging detections to date have taken place 
largely in special circumstances of external illumination, edge-on orientation, or large central clearings. At the contrast levels accessible to HST, JWST, and ground-based AO, numerous disks are unseen, perhaps because of dust grain evolution or settling which can produce self-shadowing in these optically thick systems. These dimmer, more evolved disks will be revealed around young stars by ACCESS. The disk structures seen will trace the disk dispersal process, possibly showing radial zones cleared by protoplanets, and measure the dust content as a function of age as the systems evolve toward main sequence debris disks.

For post-main sequence stars, ACCESS will image the shell structures that trace the mass-loss taking place in the Red Giant and Asymptotic Giant Branch regions of the HR diagram. The effect of low-mass companions and pre-existing debris disks on these outflows is thought to be very important for defining the variety of structures seen in the subsequent planetary nebula phase. These outflows seed the ISM with processed gas and dust leading to the next generation of stars and planetary systems. Furthermore, the large luminosity increases of a Red Giant star $(\sim 30-100$ Lsun) should lead to evaporation and disintegration of any Kuiper belt that was present during the prior main sequence phase, producing a "reborn" debris disk around first ascent red giants and possibly enabling direct imaging of extrasolar comets.

\section{OBSERVATORY ARCHITECTURE}

The ACCESS observatory is representative of the "best available" for exoplanet coronagraphy within the scope (cost, risk, schedule) of a NASA medium-class mission. We define a medium-class observatory that supports the ACCESS science objectives with mature high-readiness technologies, thus providing the basis for reliable estimates of engineering performance and science capabilities, as well as reliable determinations of cost and schedule. As indicated in Figure 3, the concept includes five major subsystems: an unobscured 1.5-meter Gregorian telescope, a spacecraft bus configured for operations at L2, a pointing control system to accurately center the star image on the coronagraph focal plane mask, an optical wavefront control system that includes two precision deformable mirrors in series, and a low-noise imaging sensor and integral-field spectrograph, all rated for a nominal 5-year science mission. We seek high confidence that all critical components of the ACCESS concept will reach TRL6 or better by the completion of Phase A. This is already the case for the ACCESS telescope and spacecraft systems. The baseline observatory architecture defines a realistic and capable platform for fair comparisons among coronagraph types.
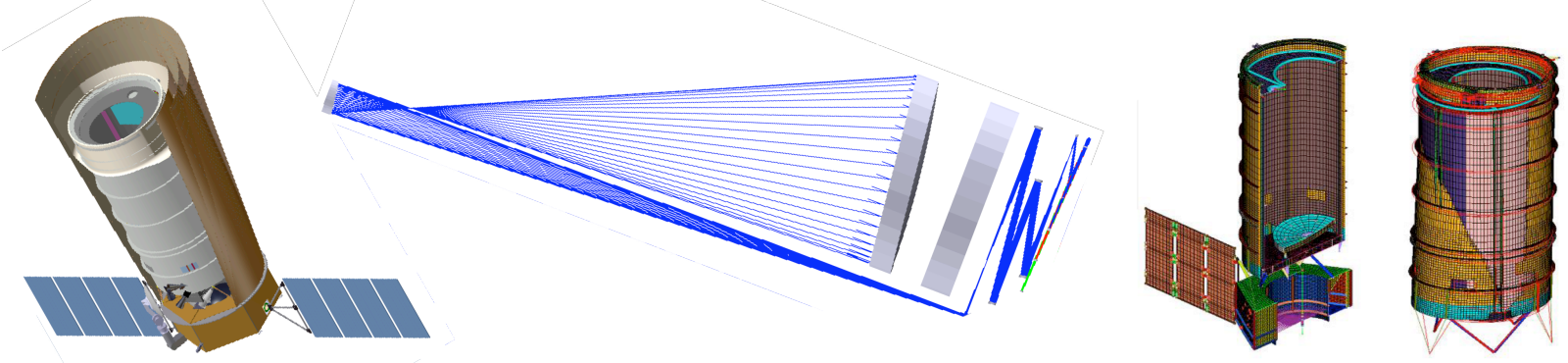

Figure 3. The ACCESS observatory. At left, a rendering of the telescope, spacecraft, multilayer sunshade, and solar panels. At center, a Zemax diagram of the off-axis Gregorian telescope, tracing the rays as they fold into the coronagraph located in the shadow of the primary mirror. At right, extensive thermal and dynamic modeling provides design guidance and reliable predictions of the optical and mechanical stability of the observatory.

Cost studies indicate that a 1.5-meter primary mirror is near the upper limit in mirror size for a medium-class mission. It will be finished to smoothness of better than $5 \mathrm{~nm} \mathrm{rms}$, similar to HST, in the critical mid-spatial-frequency (5$50 \mathrm{~cm} /$ cycle) range that scatters light within the first few arcseconds of the star. The Gregorian telescope provides a focal point between the primary and secondary mirrors, an advantageous location to place a baffle to help isolate the secondary mirror and the rest of the optical system from stray light and external thermal inputs. The secondary mirror assembly is supported by a composite forward metering shell. A temperature-controlled outer barrel assembly surrounds the entire assembly to provide thermal stability. 
The spacecraft provides payload platforms, equipment mounting panels, solar arrays, star trackers, propulsion modules, and thermal control elements. The attitude of the spacecraft is maintained by reaction wheels, using a hierarchy of knowledge from a coarse sun sensor, a pair of star trackers, and pointing knowledge from the coronagraph. A propulsion system is provided for L2 orbit insertion, station keeping, reaction wheel momentum dumping, and attitude control during contingency operations. The temperature of the payload and spacecraft components is maintained by a thermal control subsystem, including a surrounding multilayer sunshade.

\subsection{Active wavefront control}

Active control of the optical wavefront is required for our desired coronagraph system. Coronagraph elements are designed to suppress the diffracted light that is present in all telescopes, in some cases achieving in principle perfect suppression. While ideal coronagraphs perform well in simulations with a mathematically perfect wavefront, it would be unrealistic to base design decisions on ideal theoretical performance alone. Light scattered by mirror surface irregularities establishes a contrast floor that cannot be overcome by coronagraphic techniques alone. As a rule of thumb, a wavefront corrected at $n$ locations across the aperture diameter (e.g. using a DM that maps $\pi n^{2} / 4$ actuators across the primary mirror) yields a contrast floor $\mathrm{C}=\pi(2 \pi \sigma)^{2} /(n \lambda)^{2}$, where $\sigma$ is the rms surface error in the DM actuator settings, assumed random and uncorrelated. For example, with $n=48$ (a total of 1810 actuators within a circular stop) for each of two DMs, $\sigma=0.075 \mathrm{~nm} \mathrm{rms} \mathrm{is} \mathrm{required} \mathrm{for} \mathrm{a} \mathrm{raw} \mathrm{instrument} \mathrm{contrast} \mathrm{C}=10^{-9}$ at a wavelength of $550 \mathrm{~nm}$. This level of control has been demonstrated in the laboratory ${ }^{7}$.

ACCESS uses a pair of deformable mirrors and control algorithms to simultaneously control wavefront amplitude and phase, creating a high-contrast dark field of view. Two wavefront sensing and control methods are used. Each method avoids the problem of non-common-path errors by using only imagery of a star at the science focal plane. The first is the Gerchberg-Saxton method, used for on-orbit telescope alignment and initial DM settings, which requires as input a set of out-of-focus images of a target star with the coronagraph elements temporarily removed from the beam. The second is the EFC method ${ }^{8,9}$, that uses deterministic settings of the DMs to probe the complex (amplitude and phase) wavefront and iteratively reduce the energy scattered within the coronagraph dark field.

The evolution of precision deformable mirror technology based on monolithic PMN electroceramic actuator arrays is illustrated in Figure 4. The mirror facesheet, made of fused silica, is polished to a nominal surface flatness of $\lambda / 100 \mathrm{rms}$. Surface figure is settable to $0.05 \mathrm{~nm} \mathrm{rms}$ and stable (open loop) to $0.01 \mathrm{~nm}$ rms over periods of 6 hours or more in a vacuum testbed environment. All DMs have been manufactured by Northrop Grumman Xinetics.

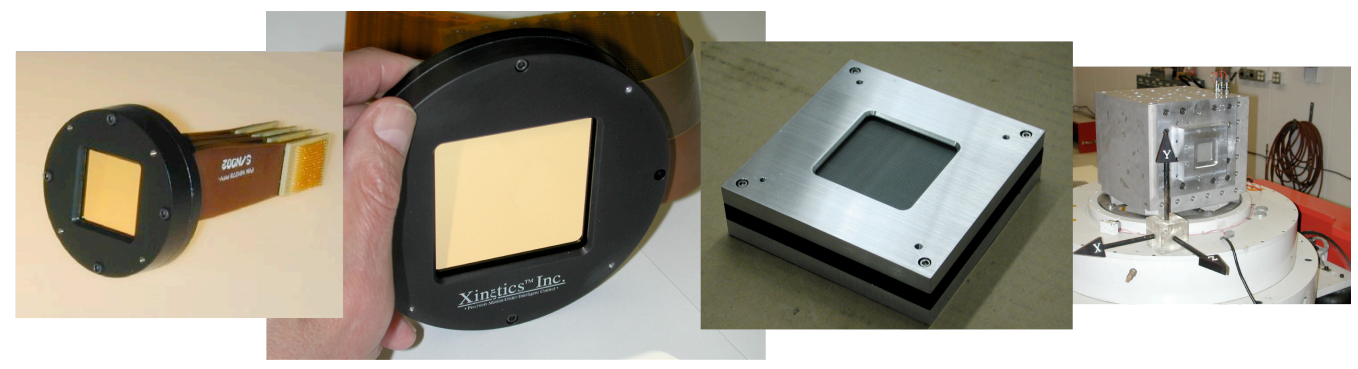

Figure 4. Development of the monolithic PMN deformable mirrors. From left to right: a $32 \times 32 \mathrm{~mm}$ array (1024 actuators) of the type used for all HCIT coronagraph contrast demonstrations to date; a $64 \times 64 \mathrm{~mm}$ array (4096 actuators) first installed on HCIT in 2009; a $48 \times 48 \mathrm{~mm}$ array (2304 actuators) to be used to demonstrate TRL6 protoflight readiness; and the $48 \times 48$ array on a shake table during environmental tests at JPL.

\subsection{The coronagraph types}

Technology readiness ranges widely for the known coronagraph types. The ACCESS study compared the performance of four representative coronagraph architectures, each subject to the predicted engineering performance of the telescope and spacecraft systems. ACCESS compared the science performance and engineering tolerances of the following four specific coronagraph methods: (1) amplitude attenuation at the focal plane (band-limited Lyot coronagraph), (2) amplitude attenuation in a pupil plane (shaped pupil coronagraph), (3) phase apodization in the pupil plane (pupil mapping or PIAA), and (4) phase modulation at the focal plane (vortex phase mask coronagraph). All four coronagraphs 
are configured for fourth-order nulls, resulting in comparable sensitivities to optical wavefront errors and misalignments. While noting that, in practice, all coronagraph types include elements to control both amplitude and phase of the complex optical wavefront, we can classify the coronagraph types by the nature and location of the dominant diffraction control element. The gamut of coronagraph types in the ACCESS study is indicated in Figure 5. A closer look reveals that the best-performing examples of each of these basic coronagraph types, far from being pure and distinct, are more usefully regarded as selected cases among a gamut of hybrid approaches. The ACCESS study used the "level playing field" provided by its baseline observatory concept to explore and quantify the advantages of the hybrid approach to coronagraph design.
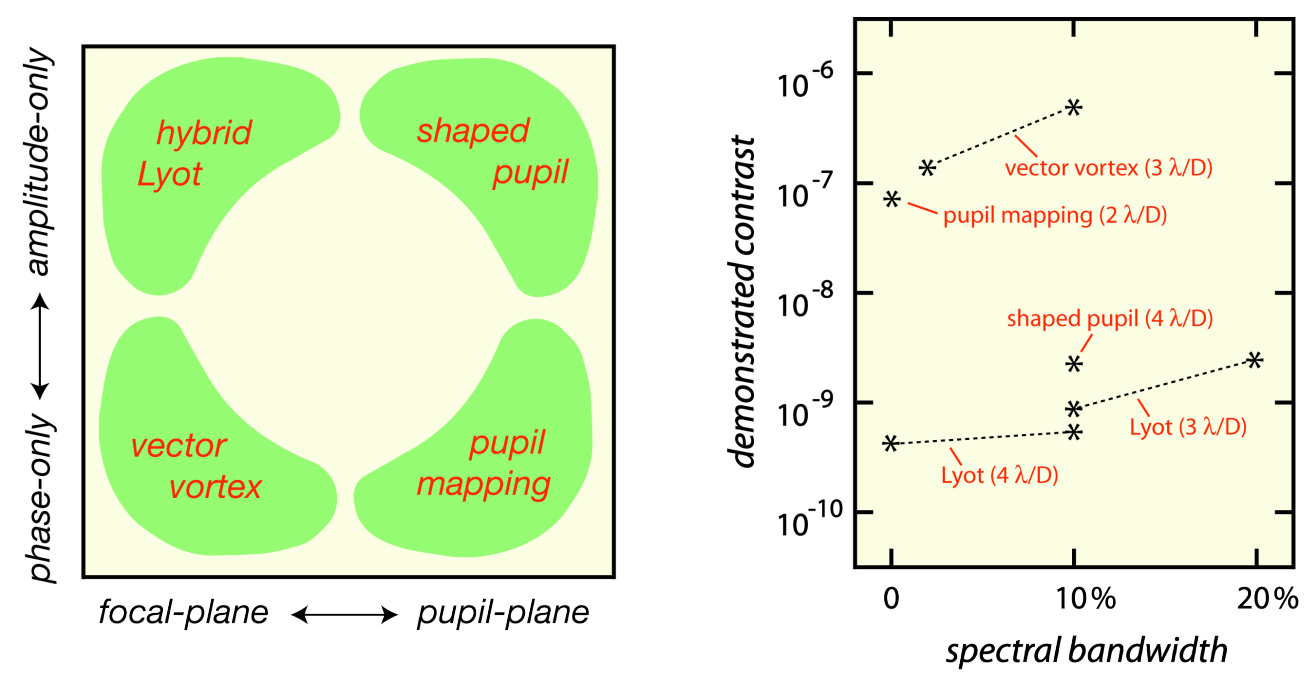

Figure 5. Left: The four coronograph types included in the ACCESS study are representative of the range of methods for internal coronagraphs. Right: The raw contrast demonstrated by these methods in the laboratory as of June 2010. See Section 3.3 in the text for details.

\subsection{Reality check: Laboratory coronagraph demonstrations}

The best demonstrated laboratory contrast to date (June 2010) for each type is plotted in Figure 5, as follows. Lyot data at $4 \lambda / \mathrm{D}$ are TPF performance milestones demonstrated on the High Contrast Imaging Testbed (HCIT) ${ }^{10,11}$ with bandlimited coronagraph masks ${ }^{12}$. Lyot data at $3 \lambda / \mathrm{D}$ were achieved on the HCIT in the course of the ACCESS study with hybrid Lyot masks ${ }^{13}$. Shaped pupil ${ }^{14}$ data were obtained on the HCIT with masks designed at Princeton ${ }^{15,16}$. The vortex results were demonstrated on the HCIT during the ACCESS study with a vector vortex mask ${ }^{17}$. The result for pupil mapping ${ }^{18,19}$ came from the NASA/Ames testbed ${ }^{20,21}$.

Further improvements in coronagraph hardware demonstrations are expected as an outcome of active research with wellunderstood technologies. Specifically, laboratory demonstrations of the hybrid Lyot coronagraph (Trauger, P.I.) and the pupil mapping coronagraph (Guyon, P.I.) are supported under NASA's Technology Development for Exoplanet Missions (TDEM) program in 2009-10. Development of the vector vortex coronagaph hardware (Serabyn, P.I.) is supported under NASA's Astronomy Research and Analysis (APRA) program for 2009-12.

Post-observation data processing methods can be expected to improve the threshold for exoplanet detection by an order of magnitude compared to the raw contrast values plotted in Figure 5, for all coronagraph types, and as illustrated in Figure 6 for the case of a Lyot coronagraph. Wavefront control and stability have been demonstrated in the laboratory at the contrast levels required to detect exoplanets. Figure 6 shows the high-contrast dark field (D-shaped) created by a single DM in laboratory experiments (a pair of DMs would clear a full, two-sided dark field). At right in the figure is a comparison of azimuthally averaged point spread functions (PSFs) of the star, with focal plane mask offset and Lyot stop in place; the coronagraph field with the DM set to a flat surface figure; the coronagraph with DM set for a dark half- 
field; and the result of simulated roll deconvolution processing with the set of 480 consecutive coronagraph images. PSFs of a standard Earth and Jupiter are also indicated for reference ${ }^{22}$.
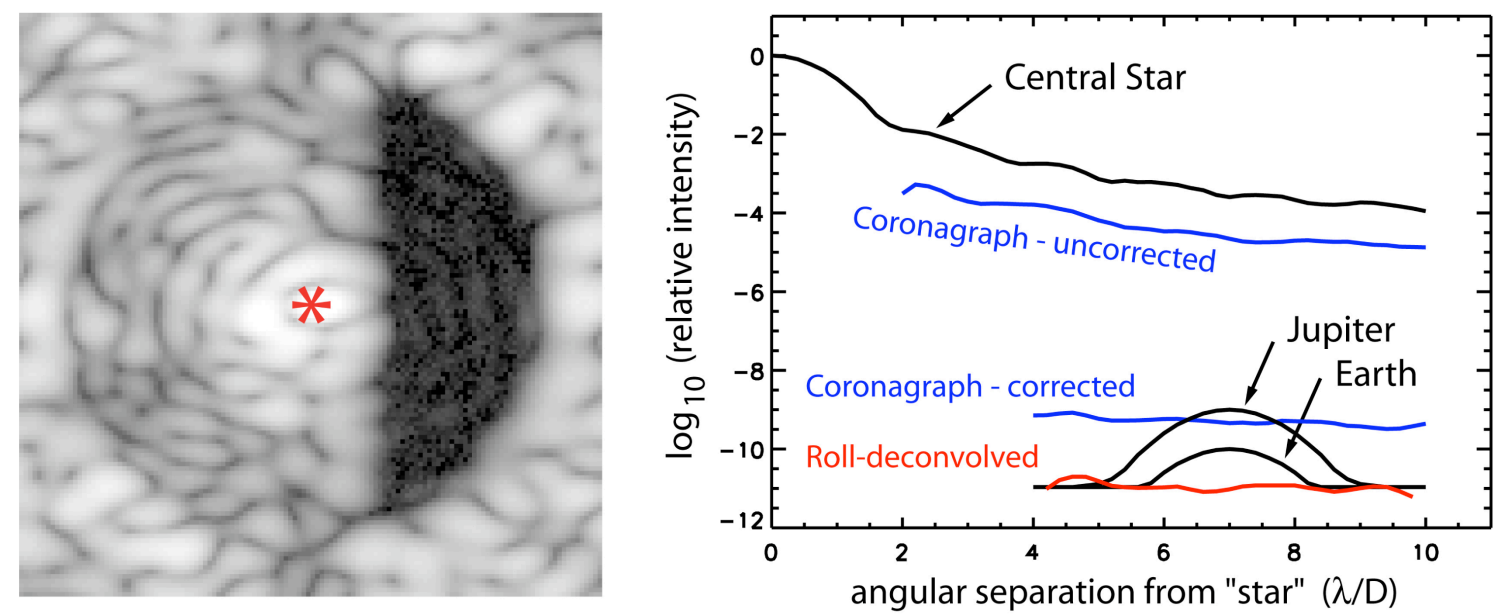

Figure 6. Left: The high-contrast dark field (D-shaped) created by a single DM in the laboratory experiments. Note that a pair of DMs, as in the ACCESS concept, creates a full two-sided dark field. Right: A comparison of the azimuthally averaged PSFs of (a) the star with focal plane mask offset and Lyot stop in place; (b) the coronograph field with all DM actuators set to equal voltages; (c) the coronograph with DM set for a dark halffield and (d) the result of simulated roll deconvolution with the set of 480 consecutive coronograph images. PSFs of a nominal Earth and Jupiter and also indicated ${ }^{22}$.

\subsection{Coronagraph optical configuration}

The major optical, structural, thermal, and mechanical elements of the observatory are common to all coronagraphs. The optical telescope assembly includes the telescope primary and secondary mirrors, a fold mirror, and a paraboloidal collimating mirror, passing a collimated beam to the instrument. The instrument receives the collimated beam and relays an image of the primary mirror (the pupil of the system) to a deformable mirror mounted in a tip/tilt fine steering mechanism. The optics to this point define the telescope line of sight (LOS). With reference to Figure 7, the beam passes to a second deformable mirror in a fixed mount, then to a paraboloidal mirror that brings the beam to focus. The image of the target star is stabilized at this focal point with a fine pointing control system using the starlight rejected by the coronagraph. Hence, all coronagraph instruments share an observatory platform that provides a stabilized telescope LOS; an internal fine guiding sensor and tip/tilt mechanism that stabilizes the star image at the first instrument focus; and a pair of high-readiness precision deformable mirrors for wavefront sensing and control.

Optical layouts for the four coronagraphs were created using Zemax raytrace code. Layouts are shown in Figure 7 for Lyot and pupil-mapping versions of the coronagraph. For clarity, only the V-band channel is shown. Corresponding layouts for the vector vortex and shaped pupil options are similar to the Lyot configuration, with different coronagraph masks inserted into the same layout of mirrors and mechanisms. The pupil-mapping option requires six additional mirrors in the optical path and two additional mechanisms. All options feature redundant assemblies of these critical elements for single-point fault tolerance: fine guidance cameras, deformable mirrors, and science cameras. The mechanical concept uses low CTE materials for dimensional stability. Temperature stability is achieved by using blankets, electrical heaters, and heat pipes. 

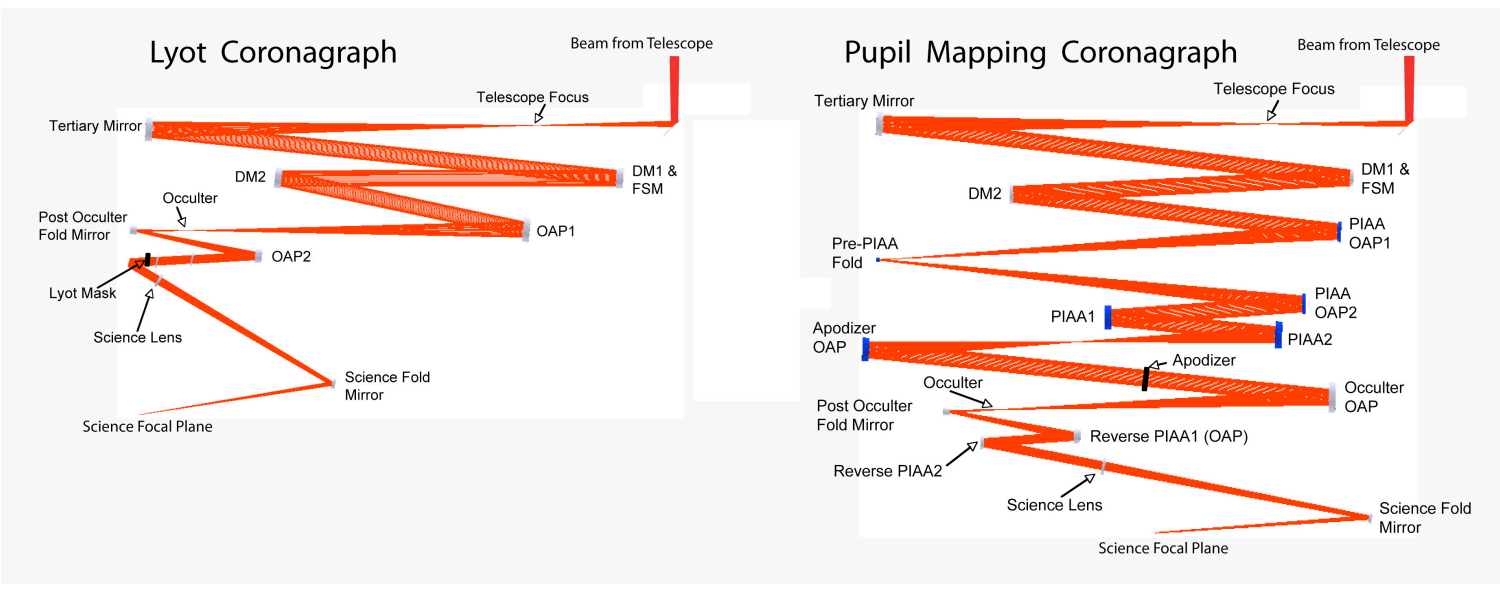

Figure 7. Optical layouts for the Lyot (at left) and pupil mapping (at right) coronagraphs. Light from the telescope enters from the upper right in each diagram, is turned by a fold mirror, passes through the telescope focal point, then to a collimating tertiary mirror. Next in the optical path is the first DM mounted in a fine steering tip/tilt flexure, followed by the second DM, then a paraboloidal mirror leading to the instrument focus. This focal point marks the location of the Lyot occulting mask, and marks the first focus in the pupil mapping optical train. The vortex and shaped pupil coronagraph layouts are nearly identical to the Lyot, with an appropriate exchange of coronagraph elements.

Each of the coronagraph types carry starlight through various optical elements to an "attenuating spot" where the bulk of the starlight is removed from the system. For the band-limited and phase mask coronagraphs, there are six critical elements between the telescope focal point and a hard-edged pupil (Lyot) stop. Similarly, there are five elements for the shaped pupil and ten elements for the pupil mapping coronagraph between telescope focal point and a hard-edged field stop. The quality and stability of these elements are critical for suppression of starlight and preservation of high contrast. The achievable contrast, inner working angle, and throughput are sensitive to surface figure errors and reflectance irregularities on these mirrors, as well as optical misalignments, telescope pointing jitter, thermal drift, scattered light from dust and surface defects, stray light, and cross-polarization effects from off-axis mirrors.

\subsection{The computational model}

Each of these model coronagraphs are integrated into the conceptual observatory platform, subject to the same telescope optical system, the same pointing and thermal control systems, and as much as possible the same end-to-end optical propagation methods. The elements of the analysis are as follows.

(1) All modeling presumes the baseline ITT telescope, NGC spacecraft, ITT/AIMS stabilization of the telescope line-ofsight, fine pointing control with the Xinetics fine steering mirror internal to the coronagraph, precision wavefront control with a pair of Xinetics $48 \times 48$ actuator DMs, JPL/ITT/NGC observatory thermal control system, and the individual instrument raytrace layouts defined by the ACCESS engineering team;

(2) Standard, realistic surface figure errors were generated for each optical element, based on measured surface PSD data for manufactured optical elements representative of the cost class for ACCESS;

(3) End-to-end Fresnel propagation (including all optical elements from star to coronagraph focal plane) is carried out with algorithms equivalent to the PROPER package ${ }^{23}$ (with one exception: simple geometric remapping was used for propagation between the PIAA coronagraph elements);

(4) DMs were used to shape the wavefront for a full "two-sided" high-contrast dark field, with settings derived using an EFC nulling algorithm and a standard model for Xinetics deformable mirror surface influence functions;

(5) The high-contrast dark field extends from the inner working angle (IWA) of the coronagraph (i.e., the angular separation from the central star beyond which the transmitted energy of a planet equals or exceeds one half of the energy in the full planet PSF) to an outer working angle (OWA) determined by the number of DM actuators mapped across the 
pupil diameter. IWA and OWA are stated in units of $\lambda_{0} / \mathrm{D}$ on the sky, where $\lambda_{0}$ is the central wavelength of the spectral band (i.e., $\lambda_{0}=540 \mathrm{~nm}$ for the $20 \% \mathrm{~V}$-band [ $\left.483-590 \mathrm{~nm}\right]$ ) and $\mathrm{D}$ is the full diameter of the telescope primary mirror;

(6) Monte Carlo simulations of pointing control dynamics, based on ACCESS analysis of telescope LOS pointing jitter ( 1 milliarcsec, $3 \sigma)$ and knowledge-limited pointing correction accuracy with the fine steering mirror $(0.45$ milliarcsec, $3 \sigma$ for a $\mathrm{V}=7$ star);

(7) Simulation of the dominant thermal response (drift in primary mirror surface figure and mutual misalignment of the primary and secondary) to a $30^{\circ}$ roll of the observatory about the line of sight to the star (with $\gamma=100^{\circ}$ ), one of several possible observing modes for discrimination between candidate exoplanets and the background field of speckles;

(8) PSFs are computed as the average of multiple (11 or more) independently-computed monochromatic PSFs distributed evenly over the $\delta \lambda / \lambda_{0}=20 \%$ wavelength band $(483-590 \mathrm{~nm})$, using known wavelength-dependent effects of the coronagraph elements; and finally,

(9) The product is a coronagraph PSF, sampled at 5 pixels per $\lambda_{0} / \mathrm{D}$ on the sky.

\subsection{Systems: Pointing control and thermal stability}

Structural and thermal models guide the observatory design and inform the optical performance models with estimates of structure dynamics, vibration isolation, pointing control, thermal gradients across the primary mirror and forward metering structures, alignment drift in response to telescope slews and roll. The required structural stability is achieved with ULE glass, Invar, and composite materials for low coefficients of thermal and moisture expansion. The relative alignment of the primary and secondary mirrors will be held stable to the 0.5 -micron level, while the surface figure of the primary mirror is held stable to the $1 \mathrm{~nm}$-rms level over the mid-spatial-frequency range.

Telescope body pointing (the LOS) is stabilized to 1 milliarcsec $(3 \sigma)$ with an active jitter control system ${ }^{24}$. Figure 8 shows the contrast deltas (vs. four specifications of rms surface quality on the optical elements following the primary mirror) due to beamwalk on the optics upstream of the fine steering mirror. The telescope attitude control system, augmented by a fine steering mirror within the coronagraph, stabilizes the star image on the coronagraph occulting mask (all four coronagraphs have a focal plane occulting mask) to 0.45 milliarcsec $(3 \sigma)^{24}$, as required for high contrast at inner working angles as small as $2 \lambda / \mathrm{D}$, as indicated in Figure 8.
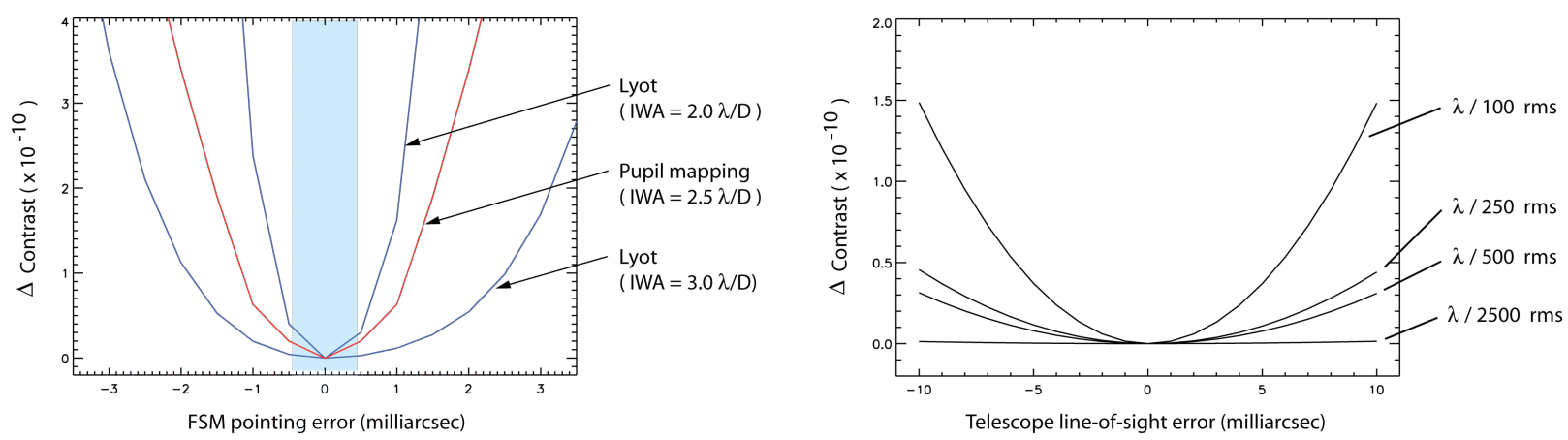

Figure 8. Left: Contrast degradation at the IWA for representative coronagraphs designed for 2.0, 2.5 and 3.0 $\lambda / \mathrm{D}$. The blue-shaded region indicates the predicted $3 \sigma$ range of pointing errors for ACCESS. Right: Contrast deltas (vs. rms surface figure of the optical elements following the primary mirror) due to beamwalk on the optics upstream of the fine steering mirror.

\subsection{Representative coronagraph for estimates of science performance}

Based on our analysis of laboratory results, given what is known and demonstrated today, we take the Lyot coronagraph as representative of the state of the art for space coronagraphs, leading to the following "science performance floor" criteria. Inner working angle is $3 \lambda_{0} / \mathrm{D}$. Spectral bandwidth is $20 \%$, which for the purpose of mission simulations will 
be centered at $540 \mathrm{~nm}$ (V-band). The coronagraph exhibits a raw contrast of $2.5 \times 10^{-9}$ averaged over $3-4 \lambda_{0} / \mathrm{D}$, as demonstrated on the HCIT during the ACCESS study, and extending to the outer working angle (OWA) $=20 \lambda_{0} / \mathrm{D}$ as defined by the ACCESS wavefront control system. These parameters establish a common point of reference for our four representative coronagraphs, anchoring the comparisons at a point in coronagraph performance trade space that corresponds to a well-defined performance floor for the ACCESS science mission. We recognize that each of the coronagraph types is capable, in principle, of exceeding these minimum requirements in one way or another. Expected advancements in performance above and beyond these minimum criteria provide the promise of a still more capable "full science mission" specific to each coronagraph type.

\subsection{Focal plane sensors and spectral discrimination}

The ACCESS focal plane will be used for simultaneous imaging in three $20 \%$ photometric bands and for integral field spectroscopy at nominal resolutions of $\mathrm{R}=20$. As noted earlier, the focal plane also provides common-path images used for telescope alignment, initial acquisition of target stars, and periodic on-orbit instrument calibration.

We anticipate that each of the coronagraph types can provide images in $20 \%$ passbands that cover the visible spectrum, e.g. $483-590 \mathrm{~nm}, 590-720 \mathrm{~nm}$, and $720-880 \mathrm{~nm}$. The high-contrast image can be split with dichroic mirrors into three spectral bands. Variations on this theme have been devised to accommodate the unique requirements of each coronagraph type. The shortest wavelength image will necessarily provide the smallest $\lambda / \mathrm{D}$ and IWA, hence the greatest sensitivity to planetary objects in the field. The longer wavelength images provide a tool for discrimination between astronomical objects and instrumental speckles in the field, and with additional integration time will provide follow-up spectrophotometry on interesting exoplanetary systems. A commercially-available low-noise CCD is used for this imaging mode.

ACCESS will include an integral field spectroscopic mode with access to all three spectral bands. A steerable mirror located just before the coronagraph focal plane and relay optics direct a selected $10 \times 10 \lambda / \mathrm{D}$ area of interest within the coronagraph dark field to a lenslet/pinhole array, followed by a diffraction grating, lenses, and a low-read-noise CCD that comprise the integral field spectrograph. The background noise, once the spectra are dispersed at $\mathrm{R}=20$, would be dominated by the read noise of conventional CCD detectors. In the last five years, electron-multiplying CCDs with gain from multi-stage electron impact ionization have been available with small but increasing formats, now up to $1 \mathrm{k} \times 1 \mathrm{k}$ pixels for the e2v L3CCD. A signal discriminator enables these detectors to become photon-counting imagers. We anticipate further advances in the area of low-noise silicon imaging detectors.

\section{SUMMARY}

The ACCESS study has considered the relative merits and readiness of four major coronagraph types in the context of a conceptual medium class space observatory. Using demonstrated high-TRL technologies, the ACCESS minimum science program surveys the nearest $120+$ AFGK stars for exoplanet systems, and surveys the majority of those for exozodiacal dust to the level of 1 zodi at 3 AU. Discoveries are followed up with R=20 spectrophotometry. Ongoing technology development and demonstrations are expected to further enhance the science reach of an ACCESS mission.

\section{ACKNOWLEDGEMENTS}

The research described in this paper was carried out at the Jet Propulsion Laboratory, California Institute of Technology, under a contract with the National Aeronautics and Space Administration.

\section{REFERENCES}

[1] Trauger, J., et al., “ACCESS Final Report,” [175 pages, copies available on request] (2009).

[2] Kalas, P., J.R. Graham, M. Clampin, "A planetary system as the origin of structure in Fomalhaut's dust belt," Nature 435, 1067 (2005).

[3] Kalas, P. et al., "Optical Images of an Exosolar Planet 25 Light-Years from Earth," Science 322, 1345 (2008).

[4] Marois, C. et al., "Direct Imaging of Multiple Planets Orbiting the Star HR 8799," Science 322, 1348 (2008).

[5] Serabyn, E. et al., "An Image of an exoplanet separated by two diffraction beamwidths from a star," Nature 464, $1018(2010)$.

[6] Lagrange, A.-M. et al., "A Giant Planet Imaged in the Disk of the Young Star bet Pictoris," Sciencexpress, 10 June 2010, $1(2010)$. 
[7] Trauger, J., et al., "Laboratory demonstrations of high-contrast imaging for space coronagraphy," Proc. SPIE 6693, 66930X (2007).

[8] Give'on, A., B. Kern, S. Shaklan, D. Moody, L. Pueyo, "Broadband wavefront correction algorithm for highcontrast imaging systems," Proc. SPIE 6691, 66910A (2007).

[9] Borde, P., and W. Traub, "High-contrast imaging from space: speckle nulling in a low aberration regime," Ap.J. $638,488(2006)$.

[10] Trauger, J., et al., “TPF-C Milestone \#1 Report,” JPL Document D-35484 (2006).

[11] Kern, B. et al., "TPF-C Milestone \#2 Report,” JPL Document D-60951 (2008).

[12] Kuchner, M.J., and W.A. Traub, "A Coronagraph with a Band-limited Mask for Finding Terrestrial Planets," Ap.J. 570,900 (2002).

[13] Moody, D., B. Gordon, and J. Trauger, "Design and demonstration of hybrid Lyot coronagraph masks for improved spectral bandwidth and throughput," Proc. SPIE 7010, 70103P (2008).

[14] Spergel, D.N., "A new pupil for detecting extra-solar planets," astro-ph/0101142 (2000).

[15] Belikov, R., et al., "Demonstration of high contrast in $10 \%$ broadband light with the shaped pupil coronagraph," Proc. SPIE, 6693, 66930Y (2007).

[16] Pueyo, L., R. Belikov, J. Kasdin, R. Vanderbei, "Performance study of integrated coronagraph-adaptive optics designs," Proc. SPIE 6693, 669307 (2007).

[17] Mawet, D., et al., "The vector vortex coronagraph: laboratory results and first light a Palomar Observatory," Astrophys. J. 709, 53 (2010).

[18] Guyon, O., et al., "Theoretical limits on extrasolar terrestrial planet detection with coronagraphs," Ap.J.Suppl. 167, 81 (2006).

[19] Guyon, O., et al., "Pupil-mapping Exoplanet Coronagraphic Observer (PECO)," Proc. SPIE 7010, 7010-66 (2008).

[20] Belikov, R., et al., "First results on a new PIAA coronagraph testbed at NASA/Ames," Proc. SPIE, 7440, 74400J (2009).

[21] Belikov, R. et al., "Laboratory demonstration of high-contrast imaging at $2 \lambda / \mathrm{D}$ enabled by temperature stabilization of the testbed," Proc. SPIE 7731, 7731-84 (2010).

[22] Trauger, J.T., and W.A. Traub, "A laboratory demonstration of the capability to image an Earth-like extrasolar planet," Nature 446, 771 (2007).

[23] Krist, J.E., "PROPER: An Optical Propagation Library for IDL," Proc. SPIE, 6675, 66750P (2007).

[24] Brugarolas, P. et al., "ACCESS pointing control system," Proc. SPIE 7731, 7731-181 (2010). 